\title{
AFFINE EQUIVALENCE AND GORENSTEINNESS
}

\author{
ANDERS FRANKILD and PETER JØRGENSEN
}

\begin{abstract}
Recently, Dwyer and Greenless established a Morita-like equivalence between categories consisting of complete modules and torsion modules. It turns out that these categories contain certain full subcategories which may be viewed as "perturbed" Auslander and Bass classes; Auslander and Bass classes are used in the study of so-called Gorenstein dimensions. This observation allows us to prove that any ideal in a commutative, local, Noetherian ring can detect whether or not the underlying ring is Gorenstein.
\end{abstract}

\section{Introduction}

\subsection{Background}

For a commutative, local, noetherian ring $R$ and an object $X$ in $\mathrm{D}(R)$, the derived category of $R$, one can consider the adjoint pair of covariant functors

$$
X \stackrel{\mathrm{L}}{\otimes}_{R}-\quad \text { and } \quad \operatorname{RHom}_{R}(X,-),
$$

and the contravariant functor

$$
\operatorname{RHom}_{R}(-, X) \text {. }
$$

It is familiar that for certain $X$ 's, these functors restrict to quasi-inverse equivalences between suitable full subcategories of $\mathrm{D}(R)$,

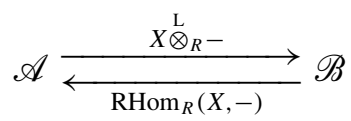

and

$$
\mathscr{C} \underset{\operatorname{RHom}_{R}(-, X)}{\stackrel{\mathrm{RHom}_{R}(-, X)}{\longleftarrow}} \mathscr{D}
$$

Important examples of this abound in the literature:

Received January 6, 2003. 


\begin{tabular}{lll}
\hline$X$ & $\begin{array}{l}\text { Equivalence theory } \\
\text { based on } \operatorname{RHom}_{R}(-, X)\end{array}$ & $\begin{array}{l}\text { Equivalence theory based on } \\
X \otimes_{R}-\text { and } \operatorname{RHom}_{R}(X,-)\end{array}$ \\
\hline$D$ & Grothendieck/Hartshorne [12] & Foxby [2] \\
$\mathrm{E}(k)$ & Matlis [14] & F+J [11] \\
$\mathrm{R} \Gamma_{\mathfrak{a}}(D)$ & Hartshorne [13] & - \\
\hline$R$ & Foxby/Yassemi [17] & Trivial \\
$\mathrm{R} \Gamma_{\mathfrak{a}}(R)$ & - & Dwyer/Greenlees [7] \\
\hline
\end{tabular}

The first three $X$ 's in the diagram are:

- $D$ is a dualizing complex for $R$.

- $\mathrm{E}(k)$ is the injective hull of $R$ 's residue class field $k$.

- $\mathrm{R} \Gamma_{\mathfrak{a}}(D)$ is obtained by taking the right derived section functor $\mathrm{R} \Gamma_{\mathfrak{a}}$ with respect to the ideal $\mathfrak{a}$ in $R$, and applying it to $D$.

The purpose of this text is to study the two theories missing from the diagram. In fact, these theories will contain the other theories in the upper right and lower left quadrants of the diagram as special cases.

\subsection{This text}

A central point of section 0.1 's diagram is that the existing equivalence theories in the upper right and lower left quadrants can recognize when the ring $R$ is Gorenstein. They do this by the sizes of the full subcategories $\mathscr{A}, \mathscr{B}, \mathscr{C}, \mathscr{D}$ in equations (0.1.1) and (0.1.2), which (in suitable senses) are maximal exactly when $R$ is Gorenstein. These results are known as "Gorenstein theorems", see [6, (2.3.14), (3.1.12), and (3.2.10)] and [11, thm. (3.5)], and live in the world of "Foxby equivalence" which deals with equivalences of categories induced by functors such as $X \stackrel{\mathrm{L}}{\otimes}{ }_{R}-$ and $\operatorname{RHom}_{R}(X,-)$, see [11].

Given this, and given that the two theories missing from section 0.1 's diagram fall in the upper right and lower left quadrants, a reasonable question is: Can the missing theories also recognize Gorenstein rings? We show in our main result, theorem 2.2, that the answer is yes. Thus, we fill in the blanks in section 0.1 's diagram by studying the missing theories and showing that they are ring theoretically interesting.

To be specific, the theories missing from section 0.1's diagram are based on the functors

$$
\mathrm{R} \Gamma_{\mathfrak{a}}(D) \stackrel{\mathrm{L}}{\otimes}{ }_{R}-\quad \text { and } \quad \mathrm{RHom}_{R}\left(\mathrm{R} \Gamma_{\mathfrak{a}}(D),-\right),
$$


respectively

$$
\operatorname{RHom}_{R}\left(-, \mathrm{R} \Gamma_{\mathfrak{a}}(R)\right),
$$

and we prove in theorem 2.2 that the subcategories between which these functors induce equivalences are maximal exactly when $R$ is Gorenstein. Note that $\operatorname{RHom}_{R}\left(-, \mathrm{R} \Gamma_{\mathfrak{a}}(R)\right)$ equals $\operatorname{RHom}_{R}(-, \mathrm{C}(\mathfrak{a}))$ where $\mathrm{C}(\mathfrak{a})$ is the Čech complex or the stable Koszul complex of a, cf. remark 1.2.

We will not reproduce theorem 2.2 in this introduction. However, in the special case $a=0$, the theorem gives corollary 2.4 which is the following improved version of the above mentioned Gorenstein theorems from [6]:

COROLLARY. Let $R$ be a commutative, local, noetherian ring with residue class field $k$. Now the following conditions are equivalent:

(1) $R$ is Gorenstein.

(2) The biduality morphism

$$
X \longrightarrow \operatorname{RHom}_{R}\left(\operatorname{RHom}_{R}(X, R), R\right)
$$

is an isomorphism for $X \in \mathrm{D}_{\mathrm{b}}^{\mathrm{f}}(R)$.

If $R$ has a dualizing complex $D$, then the above conditions are also equivalent to:

(3) $k \in \mathscr{A}_{D}$.

(4) $\mathscr{A}_{D}=\mathrm{D}(R)$.

(5) $k \in \mathscr{B}_{D}$.

(6) $\mathscr{B}_{D}=\mathrm{D}(R)$.

The notation employed here is: $\mathrm{D}_{\mathrm{b}}^{\mathrm{f}}(R)$ is the derived category of bounded complexes with finitely generated homology, and $\mathscr{A}_{D}$ and $\mathscr{B}_{D}$ are the socalled Auslander and Bass classes of $D$ which are, in a sense, the largest full subcategories of $\mathrm{D}(R)$ between which $D \stackrel{\mathrm{L}}{\otimes}{ }_{R}-$ and $\operatorname{RHom}_{R}(D,-)$ induce equivalences. See $[11,(1.5)]$ (or paragraph 1.1 below with $a=0$ ) for the technical definition of $\mathscr{A}_{D}$ and $\mathscr{B}_{D}$.

Another special case of theorem 2.2 is $\mathfrak{a}=\mathfrak{m}$ where $\mathfrak{m}$ is $R$ 's maximal ideal; this is given in corollary 2.6 which contains the Gorenstein theorem [11, thm. (3.5)]. The corollary states the following:

COROLlARY. Let $R$ be a commutative, local, noetherian ring with maximal ideal $\mathfrak{m}$ and residue class field $k=R / \mathfrak{m}$, and let $\mathrm{C}(\mathfrak{m})$ be the Čech complex of $\mathrm{m}$. Now the following conditions are equivalent:

(1) $R$ is Gorenstein. 
(2) The standard morphism

$$
\begin{aligned}
& X \stackrel{\mathrm{L}}{\otimes}{ }_{R} \mathrm{RHom}_{R}(\mathrm{C}(\mathfrak{m}), \mathrm{C}(\mathfrak{m})) \longrightarrow \mathrm{RHom}_{R}\left(\operatorname{RHom}_{R}(X, \mathrm{C}(\mathfrak{m})), \mathrm{C}(\mathfrak{m})\right) \\
& \text { is an isomorphism for } X \in \mathrm{D}_{\mathrm{b}}^{\mathrm{f}}(R) \text {. }
\end{aligned}
$$

If $R$ has a dualizing complex $D$, and $\mathrm{E}(k)$ denotes the injective hull of $k$, then the above conditions are also equivalent to:

(3) $k \in \mathscr{A}_{\mathrm{E}(k)}$.

(4) $\mathscr{A}_{\mathrm{E}(k)}=\mathbf{A}_{\mathrm{comp}}^{\mathrm{m}}$.

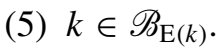

(6) $\mathscr{B}_{\mathrm{E}(k)}=\mathbf{A}_{\mathfrak{m}}^{\text {tors }}$.

Here $\mathscr{A}_{\mathrm{E}(k)}$ and $\mathscr{B}_{\mathrm{E}(k)}$ are the Auslander and Bass classes of $\mathrm{E}(k)$ which are defined in a way analogous to $\mathscr{A}_{D}$ and $\mathscr{B}_{D}$ above, see $[11,(3.3)]$, and $\mathbf{A}_{\text {comp }}^{\mathrm{m}}$ and $\mathbf{A}_{\mathfrak{m}}^{\text {tors }}$ are the categories of so-called derived complete and derived torsion complexes with respect to $\mathfrak{m}$, see [7] or remark 1.2 below.

Observe that part (2) of the corollary gives a new, simple way of characterizing Gorenstein rings. In fact, $\mathrm{RHom}_{R}(\mathrm{C}(\mathfrak{m}), \mathrm{C}(\mathfrak{m}))$ is $\widehat{R}$, the $\mathrm{m}$-adic completion of $R$, by lemma 1.9 , so part (2) of the corollary is even simpler than it first appears.

\subsection{Remarks}

The title of this text is chosen for the following reason: Hartshorne in [13] considers an instance of the contravariant equivalence theory based on $\mathrm{R} \Gamma_{\mathfrak{a}}(D)$, that is, on the functor $\operatorname{RHom}_{R}\left(-, \mathrm{R} \Gamma_{\mathfrak{a}}(D)\right)$. He calls it "affine duality". It hence seems natural that we should call the covariant equivalence theory based on $\mathrm{R} \Gamma_{\mathfrak{a}}(D)$, that is, on the functors from $(0.2 .1)$, "affine equivalence", whence our title.

Note that the equivalence theories based on the functors (0.2.1) and (0.2.2) contain a number of the other theories in section 0.1 's diagram as special cases: When $R$ has a dualizing complex $D$, the theories with $X=D$ and $X=\mathrm{E}(k)$ in the upper portion of the diagram can be obtained from the theories with $X=\mathrm{R} \Gamma_{\mathfrak{a}}(D)$; namely, $D \cong \mathrm{R} \Gamma_{0}(D)$ and $\mathrm{E}(k) \cong \mathrm{R} \Gamma_{\mathfrak{m}}(D)$. Similarly, the theories with $X=R$ in the lower portion of the diagram can be obtained from the theories with $X=\mathrm{R} \Gamma_{\mathfrak{a}}(R)$; namely, $R \cong \mathrm{R} \Gamma_{0}(R)$. Of course, this is the reason theorem 2.2 contains as a special case corollary 2.4 . 


\subsection{Synopsis}

The text is organized as follows: After this introduction comes section 1 which gives a number of ways of characterizing Gorenstein rings, plus a number of results about the derived section and completion functors, $\mathrm{R} \Gamma_{\mathfrak{a}}$ and $\mathrm{L} \Lambda^{\mathfrak{a}}$. Finally comes section 2 which gives our main result, theorem 2.2 , and concludes with some special cases in corollaries 2.4 and 2.6.

\subsection{Notation}

First note that all our results are formulated in the derived category, $\mathrm{D}(R)$. We use the hyperhomological notation set up in [9, sec. 2], with a single exception:

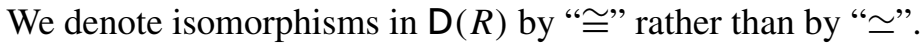

One very important tool is a number of so-called standard homomorphisms between derived functors. These are treated in [9, sec. 2], and another reference is $[6,(\mathrm{~A} .4)]$.

Apart from the material covered in [9, sec. 2], we make extensive use of the right derived section functor $\mathrm{R} \Gamma_{\mathfrak{a}}$ and the left derived completion functor $\mathrm{L} \Lambda^{\mathfrak{a}}$. They are defined as follows:

When $a$ is an ideal in $R$, the section functor with respect to $a$ is defined on modules by

$$
\Gamma_{\mathfrak{a}}(-)=\underset{n}{\operatorname{colim}} \operatorname{Hom}_{R}\left(R / \mathfrak{a}^{n},-\right)
$$

It is left exact, and has a right derived functor $\mathrm{R} \Gamma_{\mathfrak{a}}$ which lives on $\mathrm{D}(R)$. Similarly, the completion functor with respect to $a$ is defined on modules by

$$
\Lambda^{\mathfrak{a}}(-)=\lim _{n}\left(R / \mathfrak{a}^{n} \otimes_{R}-\right) .
$$

It has a left derived functor $\mathrm{L} \Lambda^{\mathfrak{a}}$ which also lives on $\mathrm{D}(R)$.

A salient fact is that $\left(\mathrm{R} \Gamma_{\mathfrak{a}}, \mathrm{L} \Lambda^{\mathfrak{a}}\right)$ is an adjoint pair. For this and other properties, see [1].

\subsection{Setup}

Throughout the text, $R$ is a commutative, local, noetherian ring with maximal ideal $\mathfrak{m}$ and residue class field $k=R / \mathfrak{m}$, and $a$ is an ideal in $R$ generated by $\boldsymbol{a}=\left(a_{1}, \ldots, a_{n}\right)$. The a-adic completion of $R$ is denoted $\widehat{R_{\mathfrak{a}}}$. The Koszul complex on $\boldsymbol{a}$ is denoted $\mathrm{K}(\boldsymbol{a})$; it is a bounded complex of finitely generated free modules. The Čech complex of $a$ (also known as the stable Koszul complex of $a$ ) is denoted $C(a)$; it is a bounded complex of flat modules. See [4, chp. 5] for a brushup on Koszul and Čech complexes. 


\section{Preparatory results}

\subsection{Affine equivalence}

Suppose that $R$ has a dualizing complex $D$. As described in the introduction, we shall consider the adjoint pair of functors

$$
\mathrm{D}(R) \underset{\mathrm{R} \Gamma_{\mathfrak{a}}(D) \stackrel{\mathrm{L}}{\otimes}_{R}-}{\stackrel{\mathrm{RHom}_{R}\left(\mathrm{R} \Gamma_{\mathfrak{a}}(D),-\right)}{\longleftarrow}} \mathrm{D}(R)
$$

Let us sum up the main content of Foxby equivalence as introduced in [11, (1.5)] in this situation: Letting $\eta$ be the unit and $\epsilon$ the counit of the adjunction, and defining the Auslander class by

$$
\mathscr{A}_{\mathrm{R} \Gamma_{\mathfrak{a}}(D)}=\left\{\begin{array}{l|l}
X & \begin{array}{l}
\eta_{X}: X \longrightarrow \mathrm{RHom}_{R}\left(\mathrm{R} \Gamma_{\mathfrak{a}}(D), \mathrm{R} \Gamma_{\mathfrak{a}}(D) \stackrel{\mathrm{L}}{\otimes}{ }_{R} X\right) \\
\text { is an isomorphism }
\end{array}
\end{array}\right\}
$$

and the Bass class by

$$
\mathscr{B}_{\mathrm{R} \Gamma_{\mathfrak{a}}(D)}=\left\{\begin{array}{l|l}
Y & \begin{array}{l}
\epsilon_{Y}: \mathrm{R} \Gamma_{\mathfrak{a}}(D) \otimes_{R} \mathrm{RHom}_{R}\left(\mathrm{R} \Gamma_{\mathfrak{a}}(D), Y\right) \longrightarrow Y \\
\text { is an isomorphism }
\end{array}
\end{array}\right\},
$$

there are quasi-inverse equivalences of categories between the Auslander and Bass classes,

$$
\mathscr{A}_{\mathrm{R} \Gamma_{\mathfrak{a}}(D)} \underset{\mathrm{R} \Gamma_{\mathfrak{a}}(D) \stackrel{\mathrm{L}}{\otimes}{ }_{R}-}{\stackrel{\mathrm{RHom}_{R}\left(\mathrm{R} \Gamma_{\mathfrak{a}}(D),-\right)}{\longleftarrow}} \mathscr{B} \Gamma_{\mathrm{R}}(D)
$$

Our main result, theorem 2.2, characterizes Gorenstein rings in terms of maximality of $\mathscr{A}_{\mathrm{R} \Gamma_{\mathfrak{a}}(D)}$ and $\mathscr{B}_{\mathrm{R} \Gamma_{\mathfrak{a}}(D)}$.

REMARK 1.2. In [7] is considered the following situation: Given a ring, $S$, and a bounded complex of finitely generated projective $S$-left-modules, $A$, one can construct the endomorphism Differential Graded Algebra, $\mathscr{E}=$ $\operatorname{Hom}_{S}(A, A)$, and $A$ becomes a Differential Graded $\mathscr{E}$-left-module whose $\mathscr{E}$-structure is compatible with its $S$-structure. Likewise, the complex $A^{\sharp}=$ $\operatorname{Hom}_{S}(A, S)$ is a bounded complex of finitely generated projective $S$-rightmodules, and becomes a Differential Graded $\mathscr{E}$-right-module whose $\mathscr{E}$-structure is compatible with its $S$-structure. Moreover, there are two full subcategories $\mathbf{A}_{\text {comp }}$ and $\mathbf{A}^{\text {tors }}$ of $\mathrm{D}(S)$, and a diagram

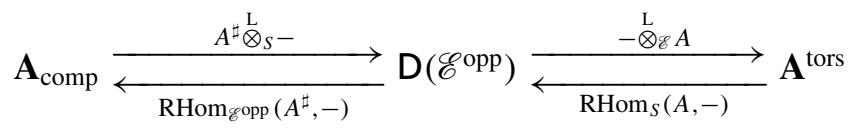


where each half is a pair of quasi-inverse equivalences of categories. Note that we write $\mathscr{E}$ opp for the opposite algebra of $\mathscr{E}$ and $\mathrm{D}(\mathscr{E}$ opp $)$ for the derived category of Differential Graded $\mathscr{E}^{\circ}$ opp-left-modules which is equivalent to the derived category of Differential Graded $\mathscr{E}$-right-modules.

In this text, we use the following special case, based on the data from setup 0.6: The ring $S$ is $R$, and the complex $A$ is $\mathrm{K}(\boldsymbol{a})$. We then write $\mathbf{A}_{\text {comp }}^{a}$ for $\mathbf{A}_{\text {comp }}$, and $\mathbf{A}_{\mathfrak{a}}^{\text {tors }}$ for $\mathbf{A}^{\text {tors }}$. By [7, proof of 4.3] and [7, prop. 6.10] we have

$$
\mathrm{K}(\boldsymbol{a})^{\sharp} \stackrel{\mathrm{L}}{\otimes_{\mathscr{E}}} \mathrm{K}(\boldsymbol{a}) \cong \operatorname{Cell}_{\mathrm{K}(\boldsymbol{a})}(R) \cong \mathrm{C}(\mathfrak{a}),
$$

so the composite of the two upper functors in diagram (1.2.1) is

$$
\begin{aligned}
& \left(\mathrm{K}(\boldsymbol{a})^{\sharp} \stackrel{\mathrm{L}}{\otimes_{R}}-\right) \stackrel{\mathrm{L}}{\otimes_{\mathscr{E}}} \mathrm{K}(\boldsymbol{a}) \simeq\left(\mathrm{K}(\boldsymbol{a})^{\sharp} \stackrel{\mathrm{L}}{\mathrm{L}_{\mathscr{E}}} \mathrm{K}(\boldsymbol{a})\right) \stackrel{\mathrm{L}}{\otimes_{R}}- \\
& \simeq \mathrm{C}(\mathfrak{a}) \stackrel{\mathrm{L}}{\otimes_{R}-},
\end{aligned}
$$

where " $\simeq$ " signifies an equivalence of functors, and where the first " $\simeq$ " is by associativity of tensor products, see [3, sec. 4.4]. Similarly, the composite of the two lower functors is

$$
\operatorname{RHom}_{\mathscr{E} \text { opp }}\left(\mathrm{K}(\boldsymbol{a})^{\sharp}, \operatorname{RHom}_{R}(\mathrm{~K}(\boldsymbol{a}),-)\right) \simeq \operatorname{RHom}_{R}\left(\mathrm{~K}(\boldsymbol{a})^{\sharp} \stackrel{\mathrm{L}}{\otimes_{\mathscr{E}}} \mathrm{K}(\boldsymbol{a}),-\right)
$$

$$
\simeq \mathrm{RHom}_{R}(\mathrm{C}(\mathfrak{a}),-) \text {, }
$$

where the first " $\simeq$ " is by adjointness, see [3, sec. 4.4]. Note that these equivalences are valid as equivalences of functors defined on the entire derived category $\mathrm{D}(R)$.

Diagram (1.2.1) shows that the essential image of the functor $\mathrm{K}(\boldsymbol{a})^{\sharp} \stackrel{\mathrm{L}}{\otimes_{R}}-$ defined on $\mathrm{D}(R)$ is all of $\mathrm{D}(\mathscr{E}$ opp $)$. (The essential image of a functor is the closure of the functor's image under isomorphisms.) In turn, equation (1.2.2) therefore shows that the essential image of the functor $\mathrm{C}(\mathfrak{a}) \stackrel{\mathrm{L}}{\otimes_{R}}-$, defined on $\mathrm{D}(R)$, equals the essential image of the functor $-\stackrel{\mathrm{L}}{\otimes_{\mathscr{E}}} \mathrm{K}(\boldsymbol{a})$, defined on all of $\mathrm{D}(\mathscr{E}$ opp $)$, and this image is $\mathbf{A}_{\mathfrak{a}}^{\text {tors }}$ by diagram (1.2.1). A similar argument with equation (1.2.3) shows that the essential image of the functor $\mathrm{RHom}_{R}(\mathrm{C}(\mathfrak{a}),-)$, defined on $\mathrm{D}(R)$, equals $\mathbf{A}_{\text {comp }}^{\mathfrak{a}}$.

Note that by $[16$, thm. $1.1(\mathrm{iv})]$ and $\left[1,(0.3)_{\text {aff }}, \mathrm{p} .4\right]$ there are natural equivalences of functors on $\mathrm{D}(R)$,

(1.2.4) $\mathrm{R} \Gamma_{\mathfrak{a}}(-) \simeq \mathrm{C}(\mathfrak{a}) \stackrel{\mathrm{L}}{\otimes}{ }_{R}-\quad$ and $\quad \mathrm{L} \Lambda^{\mathfrak{a}}(-) \simeq \mathrm{RHom}_{R}(\mathrm{C}(\mathfrak{a}),-)$,

so the above can also be phrased: The essential image of $R \Gamma_{\mathfrak{a}}$ is $\mathbf{A}_{\mathfrak{a}}^{\text {tors }}$, and the essential image of $\mathrm{L} \Lambda^{\mathfrak{a}}$ is $\mathbf{A}_{\text {comp }}^{\mathfrak{a}}$. 
Note also the following special case of the first of equations (1.2.4),

$$
\mathrm{R} \Gamma_{\mathfrak{a}}(R) \cong \mathrm{C}(\mathfrak{a}) \stackrel{\mathrm{L}}{\otimes_{R}} R \cong \mathrm{C}(\mathfrak{a}) .
$$

Computations (1.2.2) and (1.2.3) also show that ignoring the middle part of diagram (1.2.1) leaves the pair of quasi-inverse equivalences of categories

$$
\mathbf{A}_{\text {comp }} \underset{\mathrm{L} \Lambda^{\mathfrak{a}}(-) \simeq \mathrm{RHom}_{R}(\mathrm{C}(\mathfrak{a}),-)}{\stackrel{\mathrm{R} \Gamma_{\mathfrak{a}}(-) \simeq \mathrm{C}(\mathfrak{a}) \stackrel{\mathrm{D}}{R}_{R}}{\longleftarrow}} \mathbf{A}_{\mathfrak{a}}^{\text {tors }}
$$

In particular, $X \in \mathbf{A}_{\text {comp }}^{\mathfrak{a}}$ gives

$$
X \stackrel{\cong}{\longrightarrow} \mathrm{L} \Lambda^{\mathfrak{a}} \mathrm{R} \Gamma_{\mathfrak{a}} X \stackrel{\cong}{\cong} \mathrm{L} \Lambda^{\mathfrak{a}} X
$$

where the first isomorphism is the unit of the adjunction in diagram (1.2.6), and the second is by [1, p. 6, cor., part (iii)]. Similarly, $Y \in \mathbf{A}_{\mathfrak{a}}^{\text {tors }}$ gives

$$
\mathrm{R} \Gamma_{\mathfrak{a}} Y \stackrel{\cong}{\cong} \Gamma_{\mathfrak{a}} \mathrm{L} \Lambda^{\mathfrak{a}} Y \stackrel{\cong}{\longrightarrow} Y
$$

where the first isomorphism is by [1, p. 6, cor., part (iv)], and the second is the counit of the adjunction in diagram (1.2.6).

Lemma 1.3. $R$ is Gorenstein if and only if $\widehat{R_{\mathfrak{a}}}$ is Gorenstein.

Proof. The canonical homomorphism $R \longrightarrow \widehat{R_{\mathfrak{a}}}$ is flat and local by [15, p. 63, (3) and (4)]. We also have

$$
\widehat{R_{\mathfrak{a}}} / \mathrm{m} \widehat{R_{\mathfrak{a}}} \cong \widehat{R_{\mathfrak{a}}} \otimes_{R} R / \mathfrak{m}=\widehat{R_{\mathfrak{a}}} \otimes_{R} k \cong k,
$$

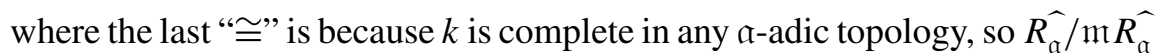
is Gorenstein. Hence $R$ and $\widehat{R_{\mathfrak{a}}}$ are Gorenstein simultaneously by [5, cor. 3.3.15].

\section{LemMa 1.4. $R$ is Gorenstein if and only if}

$$
\mathrm{RHom}_{R}\left(\operatorname{RHom}_{R}(k, R), R\right) \cong k .
$$

Proof. If $R$ is Gorenstein, then we have $\operatorname{RHom}_{R}\left(\operatorname{RHom}_{R}(k, R), R\right) \cong k$ via the biduality morphism, see [6, thm. (2.3.14)].

Conversely, suppose that (1.4.1) holds. It is easy to see in general that $\mathrm{RHom}_{R}(k, R)$ can be represented by a complex where the modules are annihilated by $\mathrm{m}$. $\operatorname{SoRHom}_{R}(k, R)$ is really just a complex over the field $k=R / \mathfrak{m}$. Hence we can use [6, (A.7.9.3)] with $V=\mathrm{RHom}_{R}(k, R)$ and $Y=R$ to get $\sup \mathrm{RHom}_{R}\left(\operatorname{RHom}_{R}(k, R), R\right)=\sup \operatorname{RHom}_{R}(k, R)-\inf \operatorname{RHom}_{R}(k, R)$. 
In the present situation, the left hand side is zero by equation (1.4.1). Hence $\sup \operatorname{RHom}_{R}(k, R)=\inf \operatorname{RHom}_{R}(k, R)$, so $\operatorname{RHom}_{R}(k, R)$ only has homology in a single degree, so only a single $\operatorname{Ext}_{R}^{i}(k, R)$ is non-zero. This implies $R$ Gorenstein by [15, thm. 18.1].

Lemma 1.5. $R$ is Gorenstein if and only if

$$
\mathrm{RHom}_{R}\left(\operatorname{RHom}_{R}(k, R), \widehat{R_{\mathfrak{a}}}\right) \cong k .
$$

in $\mathrm{D}\left(\widehat{R_{\mathfrak{a}}}\right)$.

Proof. We start with a computation in $\mathrm{D}\left(\widehat{R_{\mathfrak{a}}}\right)$,

$$
\begin{aligned}
& \mathrm{RHom}_{\hat{R}_{\mathfrak{\alpha}}^{-}}\left(\mathrm{RHom}_{R_{\mathfrak{a}}^{-}}\left(k, \widehat{R_{\mathfrak{a}}}\right), \widehat{R_{\mathfrak{a}}}\right) \\
& \stackrel{(\mathrm{a})}{\cong} \mathrm{RHom}_{R_{\mathfrak{\alpha}}^{-}}\left(\mathrm{RHom}_{R_{\mathfrak{\alpha}}^{-}}\left(k \stackrel{\mathrm{L}}{\otimes_{R}} \widehat{\widehat{R_{\mathfrak{a}}}}, \widehat{R_{\mathfrak{a}}}\right), \widehat{R_{\mathfrak{a}}}\right) \\
& \stackrel{(\mathrm{b})}{\cong} \operatorname{RHom}_{R_{\mathfrak{a}}^{-}}\left(\operatorname{RHom}_{R}\left(k, \operatorname{RHom}_{R_{\mathfrak{a}}^{-}}\left(\widehat{R_{\mathfrak{a}}}, \widehat{R_{\mathfrak{a}}}\right)\right), \widehat{R_{\mathfrak{a}}}\right) \\
& \cong \operatorname{RHom}_{R_{\mathfrak{a}}^{-}}\left(\operatorname{RHom}_{R}\left(k, \widehat{R_{\mathfrak{a}}}\right), \widehat{R_{\mathfrak{a}}}\right) \\
& \cong \operatorname{RHom}_{R_{\mathfrak{a}}^{-}}\left(\operatorname{RHom}_{R}\left(k, R \stackrel{\mathrm{L}}{\otimes_{R}} \widehat{R_{\mathfrak{a}}}\right), \widehat{R_{\mathfrak{a}}}\right) \\
& \left.\stackrel{(\mathrm{c})}{\cong} \operatorname{RHom}_{R_{\mathfrak{\alpha}}^{-}} \operatorname{RHom}_{R}(k, R) \stackrel{\mathrm{L}}{\otimes_{R}} \widehat{R_{\mathfrak{a}}}, \widehat{R_{\mathfrak{a}}}\right) \\
& \stackrel{(\mathrm{d})}{\cong} \operatorname{RHom}_{R}\left(\operatorname{RHom}_{R}(k, R), \operatorname{RHom}_{R_{\mathfrak{a}}^{-}}\left(\widehat{R_{\mathfrak{a}}}, \widehat{R_{\mathfrak{a}}}\right)\right) \\
& \cong \mathrm{RHom}_{R}\left(\operatorname{RHom}_{R}(k, R), \widehat{R_{\mathfrak{a}}}\right) \text {. }
\end{aligned}
$$

Here "(a)" is because $k \stackrel{\mathrm{L}}{\otimes_{R}} \widehat{R_{\mathrm{\alpha}}}$ is $\widehat{k_{\mathrm{a}}}$ which is just $k$ since $k$ is complete in any a-adic topology. "(b)" and "(d)" are by adjointness, [6, (A.4.21)]. "(c)" is by [6, (A.4.23)] because we have $k \in \mathrm{D}_{\mathrm{b}}^{\mathrm{f}}(R)$ and $R \in \mathrm{D}_{\mathrm{b}}(R)$, while $\widehat{R_{\mathrm{a}}}$ is a bounded complex of flat modules. Observe that both "(b)", "(c)", and "(d)" are proved using the standard homomorphisms mentioned in the introduction. The remaining isomorphisms follow from

$$
\operatorname{RHom}_{R_{\mathfrak{a}}}\left(\hat{R_{\mathfrak{a}}}, \hat{R_{\mathfrak{a}}}\right) \cong \widehat{R_{\mathfrak{a}}} \quad \text { and } \quad R \stackrel{\mathrm{L}}{\otimes_{R}} \widehat{\widehat{R_{\mathfrak{a}}}} \cong \widehat{R_{\mathfrak{a}}} \text {. }
$$

Now, $R$ is Gorenstein if and only if $\widehat{R_{\mathfrak{a}}}$ is Gorenstein by lemma 1.3. By lemma 1.4 applied to $\widehat{R_{\mathfrak{a}}}$ this amounts to

$$
\mathrm{RHom}_{R_{\mathfrak{a}}^{-}}\left(\operatorname{RHom}_{R_{\mathfrak{a}}^{-}}\left(k, \widehat{R_{\mathfrak{a}}}\right), \widehat{R_{\mathfrak{a}}}\right) \cong k .
$$


And by the above computation, this is equivalent to

$$
\operatorname{RHom}_{R}\left(\operatorname{RHom}_{R}(k, R), \widehat{R_{\mathfrak{a}}}\right) \cong k
$$

in $\mathrm{D}\left(\widehat{R_{\mathrm{a}}}\right)$.

Proposition 1.6. If $R$ has a dualizing complex $D$, then

$$
\mathscr{A}_{\mathrm{R} \Gamma_{\mathfrak{a}}(D)} \subseteq \mathbf{A}_{\text {comp }}^{\mathfrak{a}} \quad \text { and } \quad \mathscr{B}_{\mathrm{R} \Gamma_{\mathfrak{a}}(D)} \subseteq \mathbf{A}_{\mathfrak{a}}^{\text {tors }}
$$

Proof. We only prove the first inclusion, as the proof of the second is similar.

Let $X \in \mathscr{A}_{\mathrm{R} \Gamma_{\mathfrak{a}}(D)}$ be given. Then $X$ is the image under $\operatorname{RHom}_{R}\left(\mathrm{R} \Gamma_{\mathfrak{a}}(D),-\right)$ of some $Y \in \mathscr{B}_{\mathrm{R} \Gamma_{\mathfrak{a}}(D)}$, by diagram (1.1.1). Hence

$$
\begin{aligned}
X & \cong \operatorname{RHom}_{R}\left(\mathrm{R} \Gamma_{\mathfrak{a}}(D), Y\right) \\
& \stackrel{(a)}{\cong} \operatorname{RHom}_{R}\left(\mathrm{C}(\mathfrak{a}) \stackrel{\mathrm{L}}{\otimes_{R}} D, Y\right) \\
& \stackrel{(\mathrm{b})}{\cong} \operatorname{RHom}_{R}\left(\mathrm{C}(\mathfrak{a}), \operatorname{RHom}_{R}(D, Y)\right) \\
& \stackrel{(\mathrm{c})}{\cong} \mathrm{L}^{\mathfrak{a}}\left(\operatorname{RHom}_{R}(D, Y)\right),
\end{aligned}
$$

where "(a)" and "(c)" are by equations (1.2.4), and where "(b)" is by adjointness, [6, (A.4.21)].

So $X$ is in the essential image of $\mathrm{L} \Lambda^{\mathfrak{a}}$, hence $X$ is in $\mathbf{A}_{\text {comp }}^{\mathfrak{a}}$ by remark 1.2.

Lemma 1.7. We have $k \in \mathbf{A}_{\text {comp }}^{\mathfrak{a}}$ and $k \in \mathbf{A}_{\mathfrak{a}}^{\text {tors }}$.

Proof. To prove the first statement, consider

$$
\mathrm{L} \Lambda^{\mathfrak{a}}(k) \cong k \stackrel{\mathrm{L}}{\otimes_{R}} \widehat{R_{\mathfrak{a}}} \cong k
$$

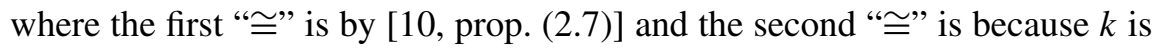
complete in any a-adic topology. This shows that $k$ is in the essential image of $\mathrm{L} \Lambda^{\mathfrak{a}}$, whence it is in $\mathbf{A}_{\text {comp }}^{\mathfrak{a}}$ by remark 1.2.

To prove the second statement, note that by [4, cor. 2.1.6] there is an injective resolution $I$ of $k$ in which each $I_{i}$ satisfies that each of its elements is annihilated by some power $\mathfrak{m}^{n}$, and hence also by some power $\mathfrak{a}^{n}$. This gives $\Gamma_{\mathfrak{a}}(I) \cong I$, and therefore

$$
\mathrm{R} \Gamma_{\mathfrak{a}}(k) \cong \Gamma_{\mathfrak{a}}(I) \cong I \cong k
$$

This shows that $k$ is in the essential image of $\mathrm{R} \Gamma_{\mathfrak{a}}$, whence it is in $\mathbf{A}_{\mathfrak{a}}^{\text {tors }}$ by remark 1.2 . 
LeMma 1.8. If $R$ has a dualizing complex $D$, then

(1) For $X \in \mathrm{D}_{+}^{\mathrm{f}}(R) \cap \mathbf{A}_{\text {comp }}^{\mathfrak{a}}$ we have

$$
\operatorname{RHom}_{R}\left(\mathrm{R} \Gamma_{\mathfrak{a}}(D), \mathrm{R} \Gamma_{\mathfrak{a}}(D) \stackrel{\mathrm{L}}{\otimes_{R}} X\right) \cong \operatorname{RHom}_{R}\left(D, D \stackrel{\mathrm{L}}{\otimes_{R}} X\right) .
$$

(2) For $Y \in D_{-}(R) \cap \mathbf{A}_{\mathfrak{a}}^{\text {tors }}$ we have

$$
\mathrm{R} \Gamma_{\mathfrak{a}}(D) \stackrel{\mathrm{L}}{\otimes_{R}} \operatorname{RHom}_{R}\left(\mathrm{R} \Gamma_{\mathfrak{a}}(D), Y\right) \cong D \stackrel{\mathrm{L}}{\otimes}{ }_{R} \operatorname{RHom}_{R}(D, Y) .
$$

Proof. We only prove (1), as the proof of (2) is similar:

$$
\begin{aligned}
& \mathrm{RHom}_{R}\left(\mathrm{R} \Gamma_{\mathfrak{a}}(D), \mathrm{R} \Gamma_{\mathfrak{a}}(D) \stackrel{\mathrm{L}}{\otimes}_{R} X\right) \\
& \quad \stackrel{(\mathrm{a})}{\cong} \operatorname{RHom}_{R}\left(\mathrm{R} \Gamma_{\mathfrak{a}}(D), \mathrm{R} \Gamma_{\mathfrak{a}}\left(D \stackrel{\mathrm{L}}{\otimes_{R}} X\right)\right) \\
& \quad \stackrel{(\mathrm{b})}{\cong} \operatorname{RHom}_{R}\left(D, \mathrm{~L} \Lambda^{\mathfrak{a}} \mathrm{R} \Gamma_{\mathfrak{a}}\left(D \stackrel{\mathrm{L}}{\otimes}_{R} X\right)\right) \\
& \quad \stackrel{(\mathrm{c})}{\cong} \operatorname{RHom}_{R}\left(D, \mathrm{~L} \Lambda^{\mathfrak{a}}\left(D \stackrel{\mathrm{L}}{\otimes_{R}} X\right)\right) \\
& \stackrel{(\mathrm{d})}{\cong} \operatorname{RHom}_{R}\left(D, D \stackrel{\mathrm{L}}{\otimes_{R}} X \stackrel{\mathrm{L}}{\otimes}_{R} \widehat{R_{\mathfrak{a}}}\right) \\
& \stackrel{(\mathrm{e})}{\cong} \operatorname{RHom}_{R}\left(D, D \stackrel{\mathrm{L}}{\otimes_{R}} \mathrm{~L} \Lambda^{\mathfrak{a}}(X)\right) \\
& \stackrel{(\mathrm{f})}{\cong} \operatorname{RHom}_{R}\left(D, D \stackrel{\mathrm{L}}{\otimes_{R}} X\right),
\end{aligned}
$$

where "(a)" follows from (1.2.4) by an easy computation, "(b)" is by [1, (0.3) aff, p. 4], "(c)" is by [1, p. 6, cor., part (iii)], "(d)" and "(e)" are by [10, prop. (2.7)], and "(f)" is by equation (1.2.7).

LEMMA 1.9. We have $\mathrm{RHom}_{R}(\mathrm{C}(\mathfrak{a}), \mathrm{C}(\mathfrak{a})) \cong \widehat{R_{\mathfrak{a}}}$ in $\mathrm{D}(R)$.

Proof. This is a computation,

$$
\begin{aligned}
\operatorname{RHom}_{R}(\mathrm{C}(\mathfrak{a}), \mathrm{C}(\mathfrak{a})) & \stackrel{(\mathfrak{a})}{\cong} \mathrm{RHom}_{R}\left(\mathrm{R} \Gamma_{\mathfrak{a}}(R), \mathrm{R} \Gamma_{\mathfrak{a}}(R)\right) \\
& \stackrel{(\mathrm{b})}{\cong} \mathrm{RHom}_{R}\left(R, \mathrm{~L} \Lambda^{\mathfrak{a}} \mathrm{R} \Gamma_{\mathfrak{a}}(R)\right) \\
& \cong \mathrm{L} \Lambda^{\mathfrak{a}} \mathrm{R} \Gamma_{\mathfrak{a}}(R) \\
& \stackrel{(\mathrm{c})}{\cong} \mathrm{L} \Lambda^{\mathfrak{a}}(R) \\
& \stackrel{(\mathrm{d})}{\cong} \bigotimes_{R} \widehat{R_{\mathfrak{a}}} \\
& \cong R_{\mathfrak{a}},
\end{aligned}
$$


where "(a)" is by equation (1.2.5), "(b)" is by [1, (0.3) aff, p. 4], "(c)" is by [1, p. 6, cor., part (iii)], and "(d)" is by [10, prop. (2.7)].

\section{The parametrized Gorenstein theorem}

Remark 2.1. Theorem 2.2 below is our main result. Among other things, it considers complexes $X$ for which the standard morphism

$$
X \stackrel{\mathrm{L}}{\otimes}{ }_{R} \operatorname{RHom}_{R}(\mathrm{C}(\mathfrak{a}), \mathrm{C}(\mathfrak{a})) \longrightarrow \mathrm{RHom}_{R}\left(\operatorname{RHom}_{R}(X, \mathrm{C}(\mathfrak{a})), \mathrm{C}(\mathfrak{a})\right)
$$

from $[6,(\mathrm{~A} .4 .24)]$ is an isomorphism. Note that by lemma 1.9 we have

$$
\mathrm{RHom}_{R}(\mathrm{C}(\mathfrak{a}), \mathrm{C}(\mathfrak{a})) \cong \widehat{R_{\mathfrak{a}}}
$$

so the $X$ 's in question have the property that there is an isomorphism

$$
X \stackrel{\mathrm{L}}{\otimes}\left(\widehat{R_{\mathfrak{a}}} \cong \operatorname{RHom}_{R}\left(\operatorname{RHom}_{R}(X, \mathrm{C}(\mathfrak{a})), \mathrm{C}(\mathfrak{a})\right) .\right.
$$

THE PARAMETRIZED GORENSTEIN THEOREM 2.2. Recall from setup 0.6 that $R$ is a commutative, local, noetherian ring which has residue class field $k$ and contains the ideal $\mathfrak{a}$, and that $\mathrm{C}(\mathfrak{a})$ denotes the Čech complex of $\mathfrak{a}$. Now the following conditions are equivalent:

(1) $R$ is Gorenstein.

(2) The standard morphism

$$
\begin{aligned}
& \quad X \stackrel{\mathrm{L}}{\otimes}{ }_{R} \operatorname{RHom}_{R}(\mathrm{C}(\mathfrak{a}), \mathrm{C}(\mathfrak{a})) \longrightarrow \mathrm{RHom}_{R}\left(\operatorname{RHom}_{R}(X, \mathrm{C}(\mathfrak{a})), \mathrm{C}(\mathfrak{a})\right) \\
& \text { is an isomorphism for } X \in \mathrm{D}_{\mathrm{b}}^{\mathrm{f}}(R) \text {. }
\end{aligned}
$$

If $R$ has a dualizing complex $D$, then the above conditions are also equivalent to the following, where we remind the reader that $\mathscr{A}_{\mathrm{R}_{\mathrm{a}}(D)}$ and $\mathscr{B}_{\mathrm{R}_{\mathrm{a}}(D)}$ were defined in paragraph 1.1:

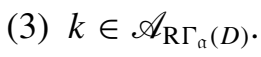

(4) $\mathscr{A}_{\mathrm{R} \Gamma_{\mathrm{a}}(D)}=\mathbf{A}_{\text {comp }}^{\mathfrak{a}}$.

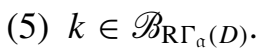

(6) $\mathscr{B}_{\mathrm{R}_{\mathfrak{a}}(D)}=\mathbf{A}_{\mathfrak{a}}^{\text {tors }}$.

Proof. We show this by showing the following implications: 
(4)

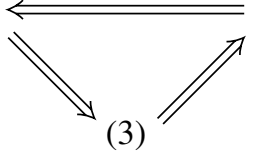

(1)

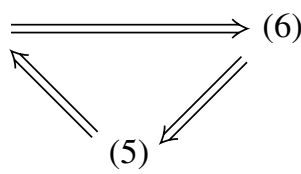

(1) $\Leftrightarrow(2)$. We start by considering the chain of morphisms

$$
\begin{aligned}
& X \stackrel{\mathrm{L}}{\otimes}{ }_{R} \operatorname{RHom}_{R}(\mathrm{C}(\mathfrak{a}), \mathrm{C}(\mathfrak{a})) \\
& \stackrel{\epsilon}{\rightarrow} \operatorname{RHom}_{R}\left(\operatorname{RHom}_{R}(X, R), R\right) \stackrel{\mathrm{L}}{\otimes}{ }_{R} \operatorname{RHom}_{R}(\mathrm{C}(\mathfrak{a}), \mathrm{C}(\mathfrak{a})) \\
& \stackrel{\alpha}{\rightarrow} \operatorname{RHom}_{R}\left(\operatorname{RHom}_{R}(X, R), R \stackrel{\mathrm{L}}{\otimes} \operatorname{RHom}_{R}(\mathrm{C}(\mathfrak{a}), \mathrm{C}(\mathfrak{a}))\right) \\
& \stackrel{\cong}{\rightrightarrows} \operatorname{RHom}_{R}\left(\operatorname{RHom}_{R}(X, R), \operatorname{RHom}_{R}(\mathrm{C}(\mathfrak{a}), \mathrm{C}(\mathfrak{a}))\right) \\
& \stackrel{\cong}{\rightleftarrows} \operatorname{RHom}_{R}\left(\operatorname{RHom}_{R}(X, R) \stackrel{\mathrm{L}}{\otimes_{R}} \mathrm{C}(\mathfrak{a}), \mathrm{C}(\mathfrak{a})\right)
\end{aligned}
$$

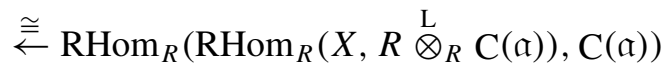

$$
\begin{aligned}
& \stackrel{\cong}{\leftarrow} \operatorname{RHom}_{R}\left(\operatorname{RHom}_{R}(X, \mathrm{C}(\mathfrak{a})), \mathrm{C}(\mathfrak{a})\right) \text {, }
\end{aligned}
$$

where $\epsilon$ is $\delta \stackrel{\mathrm{L}}{\otimes}{ }_{R} 1_{\mathrm{RHom}}(\mathrm{C}(\mathfrak{a}), \mathrm{C}(\mathfrak{a}))$ with

$$
X \stackrel{\delta}{\longrightarrow} \operatorname{RHom}_{R}\left(\operatorname{RHom}_{R}(X, R), R\right)
$$

being the biduality morphism from [6, def. (2.1.3)], and where the other arrows are either induced by the standard morphisms from [6, sec. (A.4)] or induced by the identifications

$$
R \stackrel{\mathrm{L}}{\otimes} \operatorname{RHom}_{R}(\mathrm{C}(\mathfrak{a}), \mathrm{C}(\mathfrak{a})) \stackrel{\cong}{\cong} \mathrm{RHom}_{R}(\mathrm{C}(\mathfrak{a}), \mathrm{C}(\mathfrak{a}))
$$

and

$$
R \stackrel{\mathrm{L}}{\otimes} \mathrm{C}(\mathfrak{a}) \stackrel{\cong}{\longrightarrow} \mathrm{C}(\mathfrak{a}) .
$$

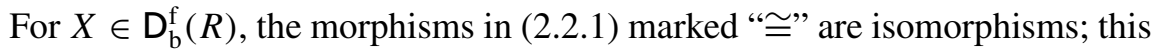
is clear except for the one second to last, for which it follows from [6, (A.4.23)] because $X \in \mathrm{D}_{\mathrm{b}}^{\mathrm{f}}(R)$ and $R \in \mathrm{D}_{\mathrm{b}}(R)$, while $\mathrm{C}(\mathfrak{a})$ is a bounded complex of flat modules. 
As one can check, the morphisms in (2.2.1) combine simply to give the standard morphism

$$
X \stackrel{\mathrm{L}}{\otimes_{R}} \operatorname{RHom}_{R}(\mathrm{C}(\mathfrak{a}), \mathrm{C}(\mathfrak{a})) \stackrel{\theta}{\longrightarrow} \operatorname{RHom}_{R}\left(\operatorname{RHom}_{R}(X, \mathrm{C}(\mathfrak{a})), \mathrm{C}(\mathfrak{a})\right)
$$

from $[6,(\mathrm{~A} .4 .24)]$.

Now suppose that (1) holds, that is, $R$ is Gorenstein, and let $X \in \mathrm{D}_{\mathrm{b}}^{\mathrm{f}}(R)$ be given. Then $\epsilon$ an isomorphism, since already the biduality morphism $\delta$ is an isomorphism [6, thm. (2.3.14)(iii')]. And $\alpha$ is an isomorphism by [6, (A.4.23)] because we have $\operatorname{RHom}_{R}(X, R) \in \mathrm{D}_{\mathrm{b}}^{\mathrm{f}}(R)$ by [6, thm. (2.3.14)(iii')], and clearly have $R \in \mathrm{D}_{\mathrm{b}}(R)$, while $\mathrm{RHom}_{R}(\mathrm{C}(\mathfrak{a}), \mathrm{C}(\mathfrak{a}))$ is isomorphic to a bounded complex of flat modules by lemma 1.9. Hence $\theta$ is an isomorphism, so (2) holds.

Conversely, suppose that (2) holds, that is, $\theta$ is an isomorphism for each $X \in \mathrm{D}_{\mathrm{b}}^{\mathrm{f}}(R)$. Letting $X$ be $k$ gives

$$
\begin{aligned}
k & \stackrel{(\mathrm{a})}{\cong} k \stackrel{\mathrm{L}}{\otimes}_{R} \widehat{R_{\mathfrak{a}}} \\
& \stackrel{(\mathrm{b})}{\cong} k \stackrel{\mathrm{L}}{\otimes}_{R} \mathrm{RHom}_{R}(\mathrm{C}(\mathfrak{a}), \mathrm{C}(\mathfrak{a})) \\
& \cong \mathrm{RHom}_{R}\left(\mathrm{RHom}_{R}(k, \mathrm{C}(\mathfrak{a})), \mathrm{C}(\mathfrak{a})\right)
\end{aligned}
$$

where "(a)" is because $k$ is complete in any a-adic topology, and "(b)" is by lemma 1.9. Now, the second half of the chain of isomorphisms (2.2.1) read backwards is

$$
\begin{aligned}
& \operatorname{RHom}_{R}\left(\operatorname{RHom}_{R}(X, \mathrm{C}(\mathfrak{a})), \mathrm{C}(\mathfrak{a})\right) \\
& \stackrel{\cong}{\rightarrow} \operatorname{RHom}_{R}\left(\operatorname{RHom}_{R}\left(X, R \stackrel{\mathrm{L}}{\otimes}{ }_{R} \mathrm{C}(\mathfrak{a})\right), \mathrm{C}(\mathfrak{a})\right) \\
& \stackrel{\cong}{\rightarrow} \operatorname{RHom}_{R}\left(\operatorname{RHom}_{R}(X, R) \stackrel{\mathrm{L}}{\otimes_{R}} \mathrm{C}(\mathfrak{a}), \mathrm{C}(\mathfrak{a})\right) \\
& \stackrel{\cong}{\rightarrow} \mathrm{RHom}_{R}\left(\mathrm{RHom}_{R}(X, R), \mathrm{RHom}_{R}(\mathrm{C}(\mathfrak{a}), \mathrm{C}(\mathfrak{a}))\right) \text {. }
\end{aligned}
$$

By lemma 1.9 we again have

$$
\begin{aligned}
& \operatorname{RHom}_{R}\left(\operatorname{RHom}_{R}(X, R), \mathrm{RHom}_{R}(\mathrm{C}(\mathfrak{a}), \mathrm{C}(\mathfrak{a}))\right) \\
& \cong \operatorname{RHom}_{R}\left(\operatorname{RHom}_{R}(X, R), \widehat{R_{\mathfrak{a}}}\right) .
\end{aligned}
$$

Setting $X=k$ and combining the three previous computations says

$$
k \cong \operatorname{RHom}_{R}\left(\operatorname{RHom}_{R}(k, R), \widehat{R_{\alpha}}\right),
$$

whence $R$ is Gorenstein by lemma 1.5 , so (1) holds. 
$(1) \Rightarrow(4)$. When $R$ is Gorenstein, then the dualizing complex $D$ is a shift of $R$ by [6, thm. (A.8.3)], so we can assume $D=R$. But then $\mathrm{R} \Gamma_{\mathfrak{a}}(D)=$ $\mathrm{R} \Gamma_{\mathfrak{a}}(R) \cong \mathrm{C}(\mathfrak{a})$ by equation (1.2.5), so the functors in diagram (1.1.1) are equivalent to the functors in diagram (1.2.6). But this certainly shows $\mathbf{A}_{\text {comp }}^{\mathfrak{a}} \subseteq$

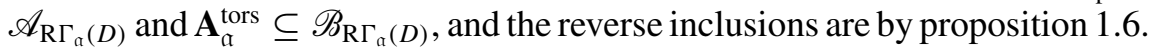

(4) $\Rightarrow$ (3). This is clear since $k \in \mathbf{A}_{\text {comp }}^{\mathfrak{a}}$ by lemma 1.7.

(3) $\Rightarrow$ (1). Suppose that $k \in \mathscr{A}_{\mathrm{R} \Gamma_{a}(D)}$ holds. It is easy to see in general that $D \stackrel{\mathrm{L}}{\otimes_{R}} k$ can be represented by a complex where all the modules are annihilated by $R$ 's maximal ideal $\mathrm{m}$. So $D \stackrel{\mathrm{L}}{\otimes_{R}} k$ is really just a complex over the field $k=R / \mathfrak{m}$, hence satisfies $\operatorname{RHom}_{k}\left(k, D \stackrel{\mathrm{L}}{\otimes_{R}} k\right) \cong D \stackrel{\mathrm{L}}{\otimes}{ }_{R} k$. This observation gives the first " $\cong$ " in

$$
\begin{aligned}
& \operatorname{RHom}_{R}\left(D, D \stackrel{\mathrm{L}}{\otimes}{ }_{R} k\right) \cong \operatorname{RHom}_{R}\left(D, \operatorname{RHom}_{k}\left(k, D \stackrel{\mathrm{L}}{\otimes}{ }_{R} k\right)\right) \\
& \cong \operatorname{RHom}_{k}(D \stackrel{\mathrm{L}}{\otimes} k, D \stackrel{\mathrm{L}}{\otimes} k) \text {, }
\end{aligned}
$$

where the second " $\cong$ ” is by adjointness, [6, (A.4.21)]. However, since $D \stackrel{\mathrm{L}}{\otimes}{ }_{R} k$ is a complex over $k$, we can use [6, (A.7.9.3)] with $V=Y=D \stackrel{\mathrm{L}}{\otimes} k$ to get

$$
\begin{aligned}
\sup \operatorname{RHom}_{k}\left(D \stackrel{\mathrm{L}}{\otimes}{ }_{R} k, D \stackrel{\mathrm{L}}{\otimes}_{R} k\right) & =\sup \operatorname{RHom}_{k}(k, D \stackrel{\mathrm{L}}{\otimes} k)-\inf \left(D \stackrel{\mathrm{L}}{\otimes}_{R} k\right) \\
& =\sup (D \stackrel{\mathrm{L}}{\otimes} k)-\inf \left(D \stackrel{\mathrm{L}}{\otimes}_{R} k\right) .
\end{aligned}
$$

Combining the equations gives

$$
\sup \operatorname{RHom}_{R}\left(D, D \stackrel{\mathrm{L}}{\otimes}{ }_{R} k\right)=\sup \left(D \stackrel{\mathrm{L}}{\otimes}{ }_{R} k\right)-\inf \left(D \stackrel{\mathrm{L}}{\otimes}{ }_{R} k\right) .
$$

Now, in the present situation, $k \in \mathscr{A}_{\mathrm{R} \Gamma_{\mathfrak{a}}(D)}$ gives the first isomorphism in

$$
\begin{aligned}
k & \stackrel{\cong}{\rightarrow} \operatorname{RHom}_{R}\left(\mathrm{R} \Gamma_{\mathfrak{a}}(D), \mathrm{R} \Gamma_{\mathfrak{a}}(D) \stackrel{\mathrm{L}}{\otimes_{R}} k\right) \\
& \cong \mathrm{RHom}_{R}\left(D, D \stackrel{\mathrm{L}}{\otimes}{ }_{R} k\right),
\end{aligned}
$$

and the second isomorphism is by lemma 1.8(1), which applies because $k \in$ $\mathbf{A}_{\text {comp }}^{\mathfrak{a}}$ by lemma 1.7. This says that the left hand side of equation (2.2.2) is zero, so $\sup \left(D \stackrel{\mathrm{L}}{\otimes_{R}} k\right)=\inf \left(D \stackrel{\mathrm{L}}{\otimes} R\right.$, so $D \stackrel{\mathrm{L}}{\otimes}{ }_{R} k$ only has homology in a single degree. By [6, eq. (A.7.4.1)] this says that $D$ has finite projective dimension, so $D$ is a non-zero complex in $\mathrm{D}_{\mathrm{b}}^{\mathrm{f}}(R)$ with finite injective and projective dimensions. Hence $R$ is Gorenstein by [8, prop. 2.10]. 
(1) $\Rightarrow(6),(6) \Rightarrow(5)$, and (5) $\Rightarrow(1)$ : These are proved by arguments dual to the ones given for (1) $\Rightarrow$ (4), (4) $\Rightarrow$ (3), and (3) $\Rightarrow$ (1).

REMARK 2.3. The reason that we refer to 2.2 as "The parametrized Gorenstein theorem" is that it is parametrized by the ideal $\mathfrak{a}$, and generalizes a number of "Gorenstein theorems" from the literature, as shown below.

COROLlary 2.4. Recall from setup 0.6 that $R$ is a commutative, local, noetherian ring with residue class field $k$. Now the following conditions are equivalent:

(1) $R$ is Gorenstein.

(2) The biduality morphism

$$
X \longrightarrow \operatorname{RHom}_{R}\left(\operatorname{RHom}_{R}(X, R), R\right)
$$

is an isomorphism for $X \in \mathrm{D}_{\mathrm{b}}^{\mathrm{f}}(R)$.

If $R$ has a dualizing complex $D$, then the above conditions are also equivalent to:

(3) $k \in \mathscr{A}_{D}$.

(4) $\mathscr{A}_{D}=\mathrm{D}(R)$.

(5) $k \in \mathscr{B}_{D}$.

(6) $\mathscr{B}_{D}=\mathrm{D}(R)$.

Proof. Immediate from theorem 2.2 by setting $\mathfrak{a}=0$.

REMARK 2.5. Note that corollary 2.4 contains several of the "Gorenstein theorems" from [6], namely, [6, (2.3.14) and (3.1.12), and (3.2.10)]. In fact, corollary 2.4 improves these results, since our classes $\mathscr{A}_{D}$ and $\mathscr{B}_{D}$ avoid the boundedness restrictions imposed in [6].

COROLlary 2.6. Recall from setup 0.6 that $R$ is a commutative, local, noetherian ring with maximal ideal $\mathrm{m}$ and residue class field $k$, and that $\mathrm{C}(\mathrm{m})$ denotes the Čech complex of $\mathrm{m}$. Now the following conditions are equivalent:

(1) $R$ is Gorenstein.

(2) The standard morphism

$$
\begin{aligned}
& X \stackrel{\mathrm{L}}{\otimes}{ }_{R} \operatorname{RHom}_{R}(\mathrm{C}(\mathfrak{m}), \mathrm{C}(\mathfrak{m})) \longrightarrow \operatorname{RHom}_{R}\left(\operatorname{RHom}_{R}(X, \mathrm{C}(\mathfrak{m})), \mathrm{C}(\mathfrak{m})\right) \\
& \text { is an isomorphism for } X \in \mathrm{D}_{\mathrm{b}}^{\mathrm{f}}(R) .
\end{aligned}
$$

If $R$ has a dualizing complex $D$, and $\mathrm{E}(k)$ denotes the injective hull of $k$, then the above conditions are also equivalent to: 
(3) $k \in \mathscr{A}_{\mathrm{E}(k)}$.

(4) $\mathscr{A}_{\mathrm{E}(k)}=\mathbf{A}_{\mathrm{comp}}^{\mathfrak{m}}$.

(5) $k \in \mathscr{B}_{\mathrm{E}(k)}$.

(6) $\mathscr{B}_{\mathrm{E}(k)}=\mathbf{A}_{\mathfrak{m}}^{\text {tors }}$.

Proof. Immediate from theorem 2.2 by setting $\mathfrak{a}=\mathfrak{m}$, since if $D$ is a dualizing complex, shifted so that its leftmost homology module sits in degree $\operatorname{dim} R$, then $\mathrm{R} \Gamma_{\mathfrak{m}}(D) \cong \mathrm{E}(k)$ by the local duality theorem, [8, p. 155].

REMARK 2.7. Note that if $R$ has a dualizing complex, then corollary 2.6 implies the "Gorenstein sensitivity theorem" [11, thm. (3.5)]. Note also that part (2) of the corollary gives a new characterization of Gorenstein rings.

\section{REFERENCES}

1. Alonso Tarrío, L., Jeremías López, A., and Lipman, J., Local homology and cohomology on schemes, Ann. Sci. École Norm. Sup. (4) 30 (1997), 1-39.

2. Avramov, L. L., and Foxby, H.-B., Ring homomorphisms and finite Gorenstein dimension, Proc. London Math. Soc. (3) 75 (1997), 241-270.

3. Avramov, L. L., and Foxby, H.-B., and Halperin, S., Differential graded homological algebra, in preparation.

4. Brodmann, M. P., and Sharp, R. Y., Local Cohomology: An Algebraic Introduction with Geometric Applications, Cambridge Stud. Adv. Math. Vol. 60, 1998.

5. Bruns, W., and Herzog, J., Cohen-Macaulay Rings, Cambridge Stud. Adv. Math. Vol. 39, 1998, revised edition.

6. Christensen, L. W., Gorenstein Dimensions, Lecture Notes in Math., Vol. 1747, 2000.

7. Dwyer, W. G., and Greenlees, J. P. C., Complete modules and torsion modules, Amer. J. Math. 124 (2002), no. 1, 199-220.

8. Foxby, H.-B., Bounded complexes of flat modules, J. Pure Appl. Algebra 15 (1979), 149-172.

9. Frankild, A., Quasi Cohen-Macaulay properties of local homomorphisms, J. Algebra 235 (2001), 214-242.

10. Frankild, A., Vanishing of local homology, Math. Z. 244 (2003), 615-630.

11. Frankild, A., and Jørgensen, P., Foxby equivalence, complete modules, and torsion modules, J. Pure Appl. Algebra 174 (2002), 135-147.

12. Grothendieck, A., Residues and Duality, Lecture Notes in Math., Vol. 20, 1966. Notes by R. Hartshorne.

13. Hartshorne, R., Affine duality and cofiniteness, Invent. Math. 9 (1970), 145-164.

14. Matlis, E., Injective modules over Noetherian rings, Pacific J. Math. 8 (1958), 511-528.

15. Matsumura, H., Commutative Ring Theory, Cambridge Stud. Adv. Math., Vol. 8, 1989, second edition. 
16. Schenzel, P., Proregular sequences, local cohomology, and completion, preprint (1998).

17. Yassemi, S., G-dimension, Math. Scand. 77 (1995), 161-174.

MATEMATISK AFDELING

KØBENHAVNS UNIVERSITET

UNIVERSITETSPARKEN 5

2100 KØBENHAVN Ø

DK-DANMARK

E-mail: frankild@math.ku.dk
DANISH NATIONAL LIBRARY OF SCIENCE AND MEDICINE NØRRE ALLÉ 49

2200 KøBENHAVN N

DK-DENMARK

E-mail:pej@dnlb.dk

CURRENT ADDRESS:

DEPARTMENT OF PURE MATHEMATICS

UNIVERSITY OF LEEDS

LEEDS LS2 9JT

UNITED KINGDOM

E-mail: popjoerg@maths.leeds.ac.uk 